\title{
Uso de encuestas en escolares para la evaluación de la cobertura y oportunidad de la vacunación en Costa Rica
}

\author{
Nidia Calvo, ${ }^{1}$ Ana Morice, ${ }^{1}$ Elizabeth Sáenz ${ }^{1}$ y Lissette Navas ${ }^{1}$
}

Forma de citar Calvo N, Morice A, Sáenz E, Navas L. Uso de encuestas en escolares para la evaluación de la cobertura y oportunidad de la vacunación en Costa Rica. Rev Panam Salud Publica. 2004;16(2):118-24.

RESUMEN Objetivo. Identificar diferencias en el nivel de cobertura y en la oportunidad de la vacunación de escolares residentes en tres tipos de zonas de Costa Rica: urbana, rural y fronteriza (rural de la frontera norte del país).

Métodos. Mediante encuesta, previa selección de escuelas por probabilidad proporcional, se reunió al azar a alumnos de primero y segundo grados de enseñanza primaria de tres zonas: urbana $(n=961)$, rural $(n=544)$ y fronteriza $(n=811)$. Los datos de las vacunas aplicadas se obtuvieron del carné (cartilla) de vacunación. Se evaluaron las diferencias en la cobertura con BCG, DPT3, VOP3, SRP1 y SRP2 y en la oportunidad de la administración de DPT1 + VOP1 antes de los 3 meses, de DPT3 + VOP3 antes de los 7 meses y de DPT4 + VOP4 + SRP1 antes de los 24 meses de edad, entre las tres zonas.

Resultados. Del total de alumnos seleccionados, $80 \%$ presentaron carné de vacunación en la zona urbana, $73 \%$ en la rural y $72 \%$ en la fronteriza $(\mathrm{P}<0.05)$. Las coberturas con BCG, DPT3, VOP3 fueron $>95 \%$ en las zonas urbana y rural, y se encontraron cifras significativamente menores ( $\mathrm{P}<0,05)$ en la zona fronteriza: $B C G, 83 \%, V O P 3,88 \%$ y DPT3, 88\%. La cobertura con SRP1 y SRP2 fue similar en las tres zonas. El porcentaje de escolares con dos o más dosis de sarampión fue: $98 \%$ en la zona urbana, 92\% en la rural y $85 \%$ en la fronteriza $(\mathrm{P}<0,05)$. Una proporción de 90\% recibió DPT1 y VOP1 antes de los tres meses de edad en la zona urbana, $89 \%$ en la rural y $80 \%$ en la fronteriza $(\mathrm{P}<0,05)$. El porcentaje de aplicación del esquema básico completo (DPT4 + VOP4 + SRP1) antes de los 24 meses fue: $93 \%$ en la zona urbana, $95 \%$ en la rural y $84 \%(\mathrm{P}<0,05)$ en la fronteriza.

Conclusiones. La zona fronteriza mostró menor cobertura y oportunidad en la aplicación de vacunas del esquema básico, con excepción de SRP. La realización de campañas de seguimiento para la erradicación del sarampión ha elevado las coberturas con la SRP1 y el refuerzo de sarampión en todas las zonas, pero el incremento ha sido mayor en la urbana. Debe hacerse un mayor esfuerzo por identificar a niños con esquemas incompletos, principalmente en zonas de alta migración.

Palabras clave Encuestas sanitarias, vacunación, migración.

Instituto Costarricense de Investigación y Enseñanza en Nutrición y Salud (INCIENSA), Tres Ríos, Costa Rica. Toda la correspondencia debe dirigirse a: Nidia Calvo, Instituto Costarricense de Investigación y Enseñanza en Nutrición y Salud. Apdo. 4-2250. Tres Ríos, Costa Rica. Correo electrónico: ncalvo@inciensa.sa.cr
Las estrategias aplicadas en Costa Rica para la prevención y control de las enfermedades inmunoprevenibles han mostrado su impacto al eliminar enfermedades como la difteria en 1976 y el tétanos neonatal en 1988. En 1994 se certificó la erradicación de la poliomielitis y desde 1999 no se registran casos de sarampión en el país. La vacunación contra hepatitis B se intro- 
dujo en 1995 y la de Haemophilus influenzae de tipo b se incorporó en 1998. La prevención del síndrome de rubéola congénita y el control acelerado de la rubéola constituyen otros objetivos importantes, para cuyo logro el gobierno nacional puso en marcha, a partir de 2001, un plan de control acelerado de la rubéola y de erradicación del sarampión (1).

A fin de sostener los logros obtenidos, Costa Rica debe mantener coberturas de vacunación mayores de 95\% en todos los distritos y cantones del país, de forma permanente y con todos los productos biológicos. Por ello, el análisis de información y la evaluación de las coberturas constituyen estrategias indispensables para fortalecer el Programa Ampliado de Inmunizaciones (PAI).

Durante el decenio de 1990, dentro del marco de la Reforma del Sector de la Salud, el país concretó cambios en la organización y funcionamiento del PAI, al fortalecer la función rectora del Ministerio de Salud y traspasar al sistema de seguridad social (Caja Costarricense de Seguro Social, CCSS) la función operativa y gerencial. Esta nueva situación creó valiosas oportunidades para el fortalecimiento del programa de inmunizaciones en el país. Favoreció una mayor cobertura de los programas de atención primaria mediante la instauración de Equipos Básicos de Atención Primaria en Salud (EBAIS) y la búsqueda de mecanismos encaminados a mejorar la eficiencia, distribución y aprovechamiento de los recursos, ente los cuales destacan los "compromisos de gestión", que incluyen objetivos e indicadores que deben cumplir los servicios de salud. En este sentido, la búsqueda de métodos que permitan mejorar el registro, la vigilancia y la evaluación de la cobertura constituye un elemento fundamental (2).

Por lo anterior, el empleo de encuestas en escolares, dado el alto índice de matrícula en la enseñanza primaria en Costa Rica, se identificó como un recurso útil para evaluar retrospectivamente las coberturas de vacunación entre 1993 y 2001, ya que permitió analizar a la cohorte de niños que en el año 2002 habían completado el esquema básico de inmunización.

Una condición metodológica que se consideró necesaria para el desarrollo del presente estudio fue que las diferencias se investigaran entre zonas con condiciones geográficas y sociales distintas, diferencias que guardan relación con la facilidad del acceso a los servicios de salud. Para ello se analizaron tres zonas: 1) zona urbana, ubicada en el área metropolitana y con mayor desarrollo social, 2) zona rural, de mediano desarrollo social $\mathrm{y}$, por último, 3) zona fronteriza (rural de la frontera norte del país), caracterizada por una elevada tasa de inmigración y bajo desarrollo social.

El objetivo de este estudió fue identificar diferencias en la oportunidad y el nivel de cobertura del esquema de inmunización con vacuna oral de polio (VOP), difteria-tos ferina-tétanos (DPT) y sarampión-rubéola-parotiditis (SRP) en escolares residentes de los tres tipos de zonas: urbana, rural y rural fronteriza en Costa Rica. El estudio permitió corroborar la utilidad del método de encuestas escolares para el análisis de coberturas, en apoyo a las estrategias de vigilancia y evaluación del programa ampliado de inmunizaciones.

\section{MATERIALES Y MÉTODOS}

\section{Población de estudio}

La población objeto de estudio estuvo constituida por todos los niños matriculados que asisten a centros educativos públicos y privados de primero y segundo grados de escuelas primarias de las siguientes zonas:

Zona 1 (rural): Ubicada en el Valle Central, está constituida por la provincia de Heredia, con sus nueve cantones: 1) Heredia (a excepción del distrito de Vara Blanca); 2) Barva; 3) Santo Domingo; 4) Santa Bárbara; 5) San Rafael; 6) San Isidro; 7) Belén; 8) Flores, y 9) San Pablo.

Zona 2 (urbana): Comprende el cantón de Santa Cruz, de la provincia de Guanacaste, conformado por nueve distritos, y el distrito de Belén, que pertenece al vecino cantón de Carrillo.

Zona 3 (rural fronteriza): Cubre el cantón de Los Chiles, localizado en el extremo norte de la provincia de Alajuela y en la zona fronteriza con Nicaragua. El cantón comprende cuatro distritos: Los Chiles, Caño Negro, El Amparo y San Jorge.

Estas tres zonas tienen características demográficas y sociales diferentes, no solo en cuanto a densidad poblacional, que es más alta en Heredia, sino también al patrón migratorio. En la primera zona la población es predominantemente urbana $(94,8 \%)$, tiene un alto índice de desarrollo social (3) (IDS $=71,4)$ y un bajo nivel de analfabetismo (1,9\%). La segunda es rural $(74,8 \%)$, tiene un desarrollo medio (IDS = 53) y un analfabetismo de 5,5\%. La tercera es rural $(85,1 \%)$, de bajo desarrollo social (IDS $=9$ ) y alto nivel de analfabetismo $(14,7 \%)$. Esta última se caracteriza por tener una alta actividad migratoria, de modo que $27,4 \%$ de su población está constituida por inmigrantes extranjeros (4) y también un alto porcentaje de inmigración nacional (23\%). En todas ellas, la proporción de la población de cinco a 14 años que acude a la escuela es superior a $85 \%$ (cuadro 1).

\section{Diseño muestral}

Como marco muestral se utilizó una lista suministrada por el Ministerio de Educación Pública, que contenía los nombres, localización y matrícula de todas las escuelas ubicadas en las zonas de interés. Con un intervalo de confianza de 95\% (IC95\%) y un error de muestreo de 3\%, el tamaño de la muestra requerido en cada una de las zonas fue de 750 sujetos en la zona urbana, 370 en la rural y 565 en la fronteriza. El número de niños evaluados se incrementó deliberadamente, en previsión de un promedio de $25 \%$ de niños sin carné de vacunación. La selección de la muestra se realizó en dos etapas. En la primera se asignó a cada escuela una probabilidad de selección proporcional a su matrícula $\mathrm{y}$, mediante 
CUADRO 1. Características sociodemográficas de zonas encuestadas en Costa Rica

\begin{tabular}{|c|c|c|c|}
\hline Características & Urbana & Rural & Fronteriza \\
\hline Extensión geográfica ${ }^{a}$ & $517 \mathrm{~km}^{2}$ & $1312 \mathrm{~km}^{2}$ & $1354 \mathrm{~km}^{2}$ \\
\hline Población total ${ }^{b}$ & 308607 & 46892 & 19732 \\
\hline Densidad poblacional (hab/km²) $)^{a, b}$ & 597 & 31 & 15 \\
\hline Población urbana ${ }^{b}$ & $76,6 \%$ & $25,2 \%$ & $14,9 \%$ \\
\hline $\begin{array}{l}\text { Índice de desarrollo social } \\
\quad \text { (intervalo: } 0 \text { a 100) }\end{array}$ & IDS = 76 & IDS = 53 & IDS $=9$ \\
\hline Analfabetismo ${ }^{b}$ & $2,3 \%$ & $5,5 \%$ & $14,7 \%$ \\
\hline $\begin{array}{l}\text { Población de } 5 \text { a } 14 \text { años } \\
\text { que asiste a la escuela }\end{array}$ & $\begin{array}{l}\text { Heredia: } 89 \% \\
\text { (urbana: } 92 \% \text {; } \\
\text { rural: } 84 \% \text { ) }\end{array}$ & $\begin{array}{l}\text { Guanacaste: } 86 \% \\
\text { (urbana: } 90 \% \text {; } \\
\text { rural: } 83 \% \text { ) }\end{array}$ & $\begin{array}{l}\text { Alajuela: } 85 \% \\
\text { (urbana: } 91 \% \text {; } \\
\text { rural: } 82 \% \text { ) }\end{array}$ \\
\hline Inmigración extranjera ${ }^{b}$ & $6,6 \%$ & $4,0 \%$ & $27,4 \%$ \\
\hline Inmigración nacional ${ }^{\mathrm{b}}$ & $41,4 \%$ & $14,0 \%$ & $23,0 \%$ \\
\hline
\end{tabular}

Fuente: Cálculos elaborados con base en:

a Costa Rica. Comisión Nacional de División Territorial Administrativa de la República de Costa Rica. San José: Imprenta Nacional; 1993.

${ }^{b}$ Costa Rica. Instituto Nacional de Estadísticas y Censos. IX censo nacional de población: características económicas. San José: INEC; 2000

${ }^{c}$ Ramírez E, Barahona M. Costa Rica: zonas de mayor y menor desarrollo relativo en Costa Rica. San José: Ministerio de Planificación y Política Económica (MIDEPLAN); 1991.

muestreo sistemático, se eligieron las escuelas que habrían de constituirse en las Unidades Primarias de Muestreo (UPM). Luego se seleccionó al azar un grupo de cada grado por escuela y, con base en la lista de estudiantes matriculados en cada grupo de los diferentes grados, por muestreo aleatorio simple se seleccionó un número de tres a 10 niños de cada grado, de acuerdo con los registros de la escuela.

\section{Recolección de datos}

Una vez aprobado el protocolo por el Comité Ético Científico Institucional, el estudio se efectuó en coordinación con las instancias pertinentes en las diferentes fases del proceso. Los datos se recabaron durante los años 2001 y 2002. Por medio de la dirección de cada escuela, se envió una nota a los padres de familia, explicándoles el estudio; se anexó una hoja de consentimiento informado para que la leyeran, firmaran si estaban de acuerdo y enviaran al niño junto con el carné de vacunación el día de la visita del equipo de investigación al recinto escolar. Los datos de identificación y domicilio fueron suministrados por el acompañante del niño o se tomaron de los registros de la dirección de la escuela. En el formulario se transcribieron las fechas de aplicación de las dosis correspondientes a cada vacuna anotada en el carné y la información se registró en una base de datos en el programa Epi Info ${ }^{\circledR} 2002$.

\section{Análisis de datos}

Mediante análisis estadístico descriptivo se caracterizaron la población y la cobertura de vacunación. Se analizaron las diferencias en las coberturas de vacunación $\left(\mathrm{IC}_{95 \%}\right)$ y se evaluaron posibles diferencias en las cohortes de grupos de edad en cuanto al esquema básico: $\mathrm{BCG}$, tres dosis de difteria, tos ferina y tétanos (DPT3), tres dosis de vacuna oral de polio (VOP3), una dosis de SRP (SRP1) y refuerzo de SRP (SRP2). Se identificaron la proporción de niños vacunados y los límites mínimo y máximo de dosis de sarampión simple (S) y de sarampión-rubéola (SR), ya que esta cohorte de niños ha sido vacunada con este tipo de producto biológico durante campañas de puesta al día y seguimiento, dentro del marco del objetivo de erradicación del sarampión en la Región de las Américas. Se estimó la oportunidad de la administración de DPT1 + VOP1 antes de los 3 meses y de DPT3 + VOP3 antes de $\operatorname{los} 7$, según la zona. Se calculó el porcentaje de cumplimiento del esquema DPT4 + VOP4 + SRP1 antes de los 24 meses de edad.

\section{RESULTADOS}

Se evaluó a 2316 alumnos de enseñanza primaria de 7 a 15 años de edad: 961 de la provincia de Heredia (zona urbana), 544 del cantón de Santa Cruz y Belén de Guanacaste (zona rural) y 811 del cantón de Los Chiles de Alajuela (zona fronteriza). En el cuadro 2 se presenta la distribución del número de escolares evaluados y con carné, por zona y grupo de edad. Una proporción de $80 \%$ (IC95\%: 77,1 a 82,3) de los niños residentes en la zona urbana presentó el carné de vacunación, $72 \%$ (IC95\%: 69,2 a 76,8) en la rural y $72 \%$ (IC95\%: 68,9 a 75,2) en la zona fronteriza.

En la distribución por zona de los niños con carné no se detectaron diferencias significativas por sexo, pero sí por edad. Asimismo, como se observa en el cuadro 2, la proporción de escolares con carné en el grupo de 6 a 8 años fue superior, aunque no en grado significativo, en las tres zonas: $86 \%$ (IC95\%: 81,8 a 90,1 ) en la urbana, $89 \%$ (IC95\%: 81,9 a 93,5 ) en la rural y $83 \%$ (IC95\%: 77,2 a 88,0) en la fronteriza. El porcentaje de niños con carné va descendiendo al aumentar la edad. Interesa señalar las diferencias en la edad de los alumnos según zona, específicamente el caso de los mayores de 12 años, o sea con "extraedad", porque este es el límite superior de edad establecido para asistir a la escuela primaria. En la zona urbana, la proporción de escolares con extraedad es de 4,3\% $(n=42)$, en la rural de $14,1 \%(n=77)$ y en la zona fronteriza de $16,7 \%(n=136)$.

No se encontraron diferencias significativas en la cobertura con BCG, DPT3 y VOP3 entre las zonas urbana y rural, y la cifra fue de $99 \%$ (cuadro 3). Sin embargo, en la zona fronteriza, las coberturas con BCG (83\%, IC $95 \%$ : 79,9 a 86,2$)$, DPT3 (88\%, IC95\%: 85,1 a 90,6) y VOP3 (88\%, IC95\%: 84,9 a 90,4) fueron significativamente inferiores a las encontradas en las otras dos zonas. 
CUADRO 2. Proporción de escolares con carné de vacunación, según grupo de edad y zona. Costa Rica, 2001-2002

\begin{tabular}{|c|c|c|c|c|c|c|c|}
\hline \multirow[b]{2}{*}{$\begin{array}{l}\text { Edad } \\
\text { (años) }\end{array}$} & \multicolumn{2}{|c|}{ Urbana } & \multicolumn{2}{|c|}{ Rural } & \multicolumn{2}{|c|}{ Fronteriza } & \multirow[b]{2}{*}{$P$} \\
\hline & $\begin{array}{l}\text { Total } \\
\text { No. }\end{array}$ & $\begin{array}{c}\text { Con carné } \\
\text { No. }(\%) \\
(\text { IC95\%) }\end{array}$ & $\begin{array}{l}\text { Total } \\
\text { No. }\end{array}$ & $\begin{array}{c}\text { Con carné } \\
\text { No. (\%) } \\
(\text { IC95\%) }\end{array}$ & $\begin{array}{l}\text { Total } \\
\text { No. }\end{array}$ & $\begin{array}{c}\text { Con carné } \\
\text { No. }(\%) \\
(\text { IC95\%) }\end{array}$ & \\
\hline 6 a 8 & 286 & $\begin{array}{c}247(86) \\
(81,8-90,1)\end{array}$ & 132 & $\begin{array}{c}117(89) \\
(81,9-93,5)\end{array}$ & 201 & $\begin{array}{c}167(83) \\
(77,2-88,0)\end{array}$ & ns \\
\hline 9 a 10 & 310 & $\begin{array}{c}256(83) \\
(77,8-86,6)\end{array}$ & 167 & $\begin{array}{c}134(80) \\
(73,4-86,0)\end{array}$ & 242 & $\begin{array}{c}175(72) \\
(66,2-77,8)\end{array}$ & $<0,05$ \\
\hline 11 a 12 & 319 & $\begin{array}{c}246(77) \\
(72,1-81,6)\end{array}$ & 167 & $\begin{array}{c}105(63) \\
(55,1-70,2)\end{array}$ & 232 & $\begin{array}{c}154(66) \\
(59,9-72,4)\end{array}$ & $<0,05$ \\
\hline 13 a 15 & 42 & $\begin{array}{c}18(43) \\
(27,7-59,0)\end{array}$ & 77 & $\begin{array}{c}42(55) \\
(42,8-65,9)\end{array}$ & 136 & $\begin{array}{c}89(65) \\
(56,8-73,4)\end{array}$ & $<0,05$ \\
\hline Total & 961 & $\begin{array}{c}767(80) \\
(77,1-82,3)\end{array}$ & 544 & $\begin{array}{c}398(73) \\
(69,2-76,8)\end{array}$ & 811 & $\begin{array}{c}585(72) \\
(68,9-75,2)\end{array}$ & $<0,05$ \\
\hline
\end{tabular}

a Intervalo de confianza de $95 \%$.

No se identificaron diferencias en las coberturas de SRP1 entre las tres zonas: 96\% (IC95\%: 94,6 a 97,4) en la urbana, 96\% (IC95\%: 94,1 a 98,0) en la rural y 94\% (IC95\%: 91,6 a 95,7) en la zona fronteriza. El porcentaje de escolares con refuerzo de SRP también mostró coberturas similares en las tres zonas: 76\% (IC95\%: 72,5 a 78,7) en la urbana, 72\% (IC95\%: 66,9 a 75,9) en la rural y 71\% (IC95\%: 67,1 a 74,6) en la fronteriza.

Sin embargo, al analizar el número de dosis de sarampión, ya sea SS, SR o SRP (cuadro 3), se encontró que 98\% (IC95\%: 96,6 a 98,8) de los escolares de la zona urbana, 92\% (IC95\%: 88,8 a 94,4) de la rural y 85\% (IC95\%: 81,8 a 87,7) de la fronteriza tenían dos o más dosis, por lo que esta cobertura fue significativamente mayor en la zona urbana. El número promedio de aplicaciones de vacuna contra el sarampión fue de 3,8 en la zona urbana, 2,5 en la rural y 2,9 en la fronteriza. El máximo de dosis contra el sarampión alcanzó cifras de siete en la zona urbana, cinco en la rural y nueve en la zona fronteriza (figura 1).

El cuadro 4 muestra algunos indicadores de oportunidad en la administración de las vacunas del esquema básico. En la zona urbana, 90\% (IC95\%:

CUADRO 3. Coberturas de esquema básico de vacunación en escolares, según zona. Costa Rica, 2001-2002

\begin{tabular}{|c|c|c|c|c|}
\hline & Urbana & Rural & Fronteriza & \\
\hline Vacuna & $\begin{array}{l}\text { No. (\%) } \\
(\text { IC95\%) }\end{array}$ & $\begin{array}{l}\text { No. (\%) } \\
(\text { IC95\%) }\end{array}$ & $\begin{array}{l}\text { No. (\%) } \\
(\text { IC95\%) }\end{array}$ & $P$ \\
\hline$B C G$ & $\begin{array}{c}760(99) \\
98,1-99,6\end{array}$ & $\begin{array}{c}395(99) \\
97,8-99,8\end{array}$ & $\begin{array}{c}487(83) \\
79,9-86,2\end{array}$ & $<0,05$ \\
\hline VOP3 & $\begin{array}{c}757(99) \\
97,6-99,4\end{array}$ & $\begin{array}{c}394(99) \\
97,8-99,8\end{array}$ & $\begin{array}{c}515(88) \\
85,1-90,6\end{array}$ & $<0,05$ \\
\hline DPT3 & $\begin{array}{c}757(99) \\
97,6-99,4\end{array}$ & $\begin{array}{c}394(99) \\
97,4-99,7\end{array}$ & $\begin{array}{c}514(88) \\
84,9-90,4\end{array}$ & $<0,05$ \\
\hline SRP1 & $\begin{array}{c}738(96) \\
94,6-97,4\end{array}$ & $\begin{array}{c}384(96) \\
94,1-98,0\end{array}$ & $\begin{array}{c}549(94) \\
91,6-95,7\end{array}$ & ns \\
\hline SRP2 & $\begin{array}{c}581(76) \\
72,5-78,7\end{array}$ & $\begin{array}{c}285(72) \\
66,9-75,9\end{array}$ & $\begin{array}{c}415(71) \\
67,1-74,6\end{array}$ & ns \\
\hline $\begin{array}{l}\text { S-SR-SRP } \\
\text { (2 dosis) }\end{array}$ & $\begin{array}{c}751(98) \\
96,6-98,8\end{array}$ & $\begin{array}{c}366(92) \\
88,8-94,4\end{array}$ & $\begin{array}{c}497(85) \\
81,8-87,7\end{array}$ & $<0,05$ \\
\hline
\end{tabular}

\footnotetext{
a Intervalo de confianza de $95 \%$.
}

88,0 a 92,2) de los niños estaban vacunados con VOP1 y DPT1 antes de los 3 meses de edad, 89\% (IC95\%: 83,6 a $91,5)$ en la zona rural y $80 \%$ (IC95\%: $76,9$ a 83,4$)$ en la zona fronteriza. Al calcular la proporción de escolares que recibieron la tercera dosis de VOP y DPT antes de los 7 meses de edad, las cifras fueron similares en las zonas urbana y rural, con $70 \%$ de los niños vacunados, pero en la zona fronteriza ese porcentaje fue de 55\% (IC95\%: 50,6 a 58,8). Con respecto a la proporción de niños con esquema básico completo (DPT4 + VOP4 + SRP1) antes de los 24 meses, se encontró un 93\% (IC95\%: 90,8 a 94,6) de cumplimiento del indicador en la zona urbana, 95\% (IC95\%: 92,0 a 96,7) en la rural y $84 \%$ (IC95\%: 81,0 a 87,1$)$ en la zona fronteriza $P<0,05)$.

\section{DISCUSIÓN}

Diversos factores relacionados con la distancia a centros de salud y otros de tipo socioeconómico relacionados con el contexto familiar, como el nivel educativo (escolaridad) y el empleo materno, se han señalado como factores vinculados al nivel de cobertura de la vacunación (5). Costa Rica es un país que se ha caracterizado por un acceso universal a la salud y una extensa red de establecimientos y servicios de la seguridad social, lo que significa que $87 \%$ de la población reside a menos de $4 \mathrm{~km}$ de distancia de un centro de atención primaria (6). Por tanto, no parece que dificultades de acceso geográfico sean un obstáculo para el logro de coberturas adecuadas en el esquema básico de vacunación en el país.

Como resultado de los esfuerzos de la reforma del sector de la salud y las estrategias para avanzar en el control y la erradicación de las enfermedades inmunoprevenibles en la Región de las Américas, las coberturas de vacunación en Costa Rica lograron una mejoría importante desde mediados del decenio de 1990. Sin embargo, se ha observado que las coberturas de vacunación notificadas oficialmente informan un subregistro que oscila en torno a $6,6 \%$ en el caso de VOP3 y de $11,5 \%$ 
FIGURA 1. Número de dosis de sarampión (SS, SR, SRP) en escolares, según zona. Costa Rica, 2001-2002

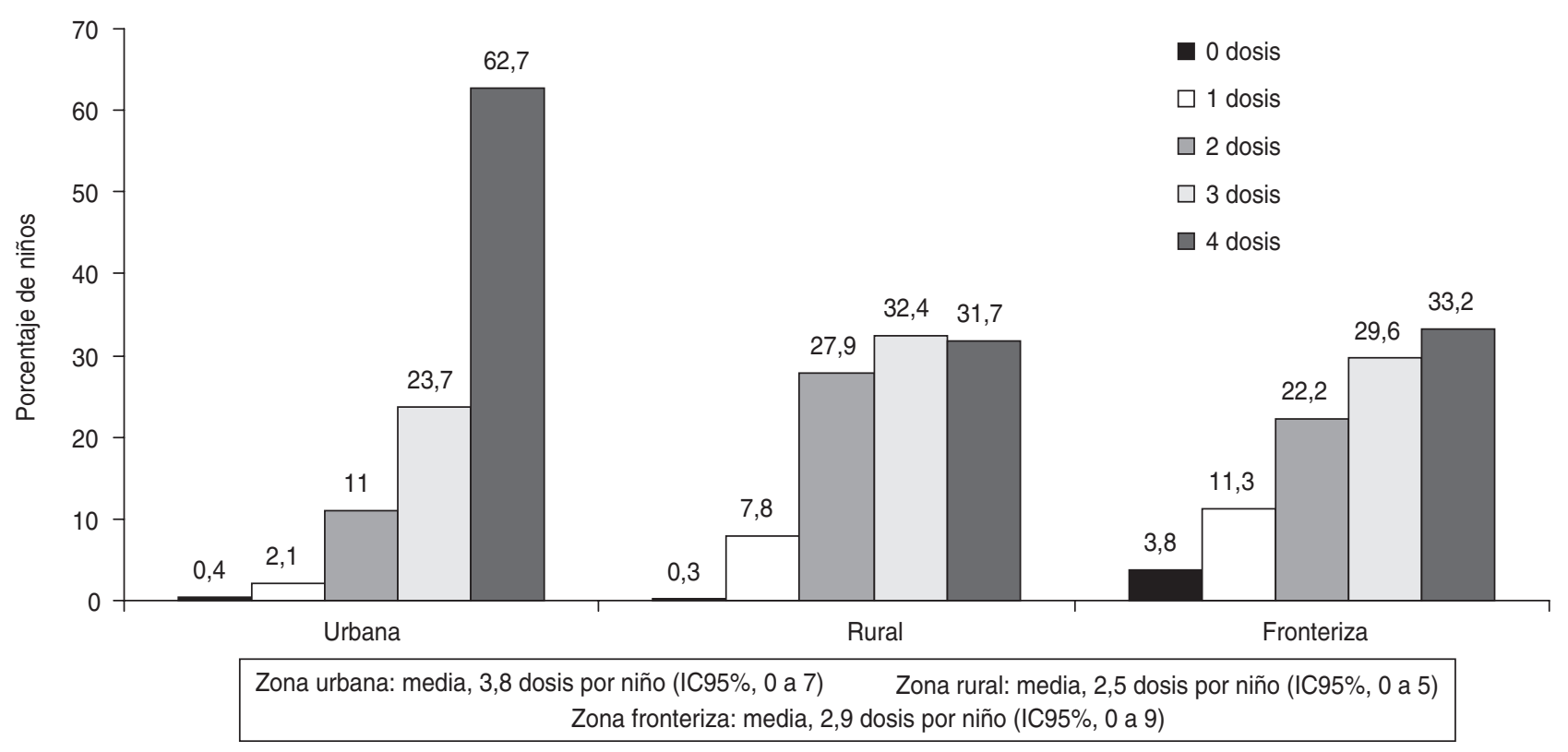

en el de SRP, el cual es superior en el área metropolitana. ${ }^{2}$

La participación de las escuelas se plantea como una valiosa estrategia para actualizar esquemas y vigilar las coberturas alcanzadas en el esquema regular de vacunación (7). Para ello, es necesario establecer reglamentos apropiados de alcance nacional, sin perder de vista que su cumplimiento adecuado depende también de factores socioeconómicos que requieren acciones eficaces de vigilancia y control.

El presente estudio no documentó diferencias importantes en la cobertura ni oportunidad de la vacunación entre las zonas urbana y rural, pero sí se identificaron rezagos en la zona fronteriza, tanto en cobertura como en oportunidad, en lo que respecta a las vacunas BCG, DPT3 y VOP3. Esas diferencias podrían explicarse en alguna medida por la alta actividad migratoria que caracteriza a la zona fronteriza, con 27,4\% de inmigración extranjera según el censo nacional de 2000, lo cual contrasta con la estabilidad poblacional de

\footnotetext{
2 Machado V, Morice A. Carrillo W. Análisis de áreas críticas de cobertura de vacunación mediante empleo de diversas fuentes de información, Costa Rica 1998-2002 [documento inédito], 2002.
}

las otras zonas, cuya proporción de inmigración extranjera es de $6,6 \%$ en la zona urbana y de $6,6 \%$ en la rural.

Otro elemento que puede explicar la menor cobertura en la frontera es la diferencia en la distribución proporcional de edad de los grupos de escolares, ya que los niños mayores de 12 años que asisten a las escuelas corresponden a una cohorte de población con menor cobertura de vacunación, si se comparan con los grupos de menor edad. Dada la mayor proporción de escolares con extraedad en la zona fronteriza $(16,7 \%)$ si se compara con la

CUADRO 4. Indicadores de la oportunidad de la aplicación de vacunas, según zona. Costa Rica, 2001-2002

\begin{tabular}{lcccc}
\hline & \multicolumn{4}{c}{ Zona } \\
\cline { 2 - 4 } & $\begin{array}{c}\text { Urbana } \\
\text { No. (\%) }\end{array}$ & $\begin{array}{c}\text { Rural } \\
\text { No. }(\%)\end{array}$ & $\begin{array}{c}\text { Fronteriza } \\
\text { No. }(\%)\end{array}$ & \\
Vacuna & $($ IC95\%) & $($ IC95\%) & \\
\hline VOP1 + DPT1 & $693(90)$ & $353(89)$ & $470(80)$ & $<0,05$ \\
antes de 3 meses & $88,0-92,2$ & $85,1-91,6$ & $76,9-83,4$ & \\
VOP3 + DPT3 & $534(70)$ & $280(70)$ & $320(55)$ & $<0,05$ \\
antes de 7 meses & $66,2-72,8$ & $65,6-74,5$ & $50,6-58,8$ & \\
DPT4 + VOP4 + SRP1 & $712(93)$ & $377(95)$ & $493(84)$ & $<0,05$ \\
antes de 24 meses & $90,8-94,6$ & $92,0-96,7$ & $81,0-87,1$ & \\
\hline
\end{tabular}

zona urbana, donde la cifra es de $4,3 \%$, se introduce un sesgo hacia un menor promedio de coberturas en la población escolar, determinado por diferencias en la distribución de edad de la población escolar.

Respecto a las coberturas con SRP1 y $\mathrm{SRP} 2$, fueron similares en las tres zonas. Es probable que este resultado se relacione con las campañas de puesta al día y seguimiento que el Estado instauró en los años 90 para lograr la erradicación del sarampión, las cuales han permitido actualizar los esquemas de vacunación con este producto biológico. 
Otro aspecto que se ha visto fortalecido con la instauración de campañas es la aplicación del segundo refuerzo de sarampión, ya que cuando la cobertura se analiza incorporando tanto la vacuna simple (SS) como las formas combinadas (SR y SRP) la cifra se eleva de 76 a $98 \%$ en la zona urbana, 72 a $92 \%$ en la rural y 71 a $85 \%$ en la fronteriza. Sin embargo, mientras que algunos niños recibieron hasta nueve dosis contra el sarampión en la zona fronteriza, 3,8\% de los niños de esa zona no recibieron ninguna dosis. Este dato pone de relieve la importancia de reforzar la identificación de bolsones de "no vacunados" en lo que se refiere tanto a la vacunación del programa ordinario como a las estrategias de vacunación masiva en campañas especiales. Por lo anterior, es necesario reforzar las acciones de vigilancia local de coberturas para poder vacunar a grupos poblacionales rezagados en la vacunación y a la población de inmigrantes que se incorporan en el sistema educativo y de salud.

Otros países se han planteado estrategias distintas para apoyar la vigilancia y la evaluación de las coberturas de vacunación (8-14). Es necesario considerar algunos requisitos si se desea aplicar encuestas entre escolares para evaluar coberturas. En primer lugar se requiere tener una matrícula escolar alta y deserción escolar baja. Otro elemento importante es que no existan diferencias socioeconómicas importantes con respecto a las posibilidades de acceso a la enseñanza primaria, de manera que se facilite la comparación entre zonas geográficas.

El uso de la cartilla o carné como fuente de información sobre la cobertura es también una condición fundamental para la evaluación de esta (15). El presente estudio demostró que cerca de $80 \%$ de los escolares portaban este documento. Se informan cifras mayores de $90 \%$ del uso del carné en niños menores de un año (16), por lo cual, el encontrar disponible el carné en poblaciones de niños de mayor edad, constituye un elemento en favor de la utilidad de este tipo de encuestas.

En el caso de Costa Rica se cumplen los requisitos señalados en los párrafos anteriores (17), por lo que este tipo de estudio aportó resultados confiables que apoyaron la identificación de in- tervenciones para reforzar el programa nacional de vacunación en el país. Entre estas acciones cabe recomendar el fortalecimiento de la revisión del carné de vacunación al ingreso en la escuela, para asegurar una mayor cobertura del segundo refuerzo de SRP, y la importancia de realizar un trabajo integrado con el sector educativo. Este tipo de evaluaciones e intervenciones deben trascender la vigilancia de alcance nacional para brindar también retroalimentación, además de realizarse desde el ámbito local, mediante un trabajo conjunto entre los servicios de salud y las escuelas.

Como recurso para la recolección de datos, las encuestas escolares tienen menor costo que las encuestas de hogares, porque utilizan una muestra de población cautiva y permiten evaluar las tendencias y cambios en el tiempo del programa de inmunizaciones en una cohorte. Por tanto, este método constituye una opción muy útil para evaluar la cobertura de vacunación. Además, la información que aporta promueve la articulación de los programas de vacunación con los de salud escolar.

\section{REFERENCIAS}

1. Morice A, Carvajal X, León M, Machado V, Badilla X, Reef S, et al. Accelerated rubella control and congenital rubella syndrome prevention strengthen measles eradication: the Costa Rican experience. J Infect Dis. 2003;187 (Suppl 1):158-63.

2. Organización Panamericana de la Salud, Ministerio de Salud, Caja Costarricense de Seguro Social. Evaluación internacional del Programa de Inmunizaciones en Costa Rica. San José, Costa Rica: Ministerio de Salud; 2000.

3. Ministerio de Planificación Nacional y Política Económica. Índice de desarrollo social. San José, Costa Rica: MIDEPLAN; 1998.

4. Instituto Nacional de Estadísticas y Censos. IX Censo Nacional de Población. Características sociales y demográficas. San José, Costa Rica: INEC; 2002.

5. Morales JC, Barradas R, Sampaio MC, Carrara P. Cobertura vacunal en el primer año de vida en cuatro ciudades del estado de São Paulo, Brasil. Rev Panam Salud Publica. 2000;8:2-18.

6. Rosero-Bixby L. Spatial access to health care in Costa Rica and its equity: a GIS-based study. Soc Sci Med. 2004;58: 1271-84.

7. Linton LS, Peddecord KM, Seidman RL, Edwards C, Ross S, Gustafson K, et al. Implement- ing a seventh grade vaccination law: school factors associated with completion of required immunizations. Prev Med. 2003;36: 510-7.

8. Chen R, Orenstein WA. Epidemiologic methods in immunization programs. Epidemiol Rev. 1996;18:99-117.

9. Expanded Programme on Immunization. Evaluation and monitoring of national immunization programmes. Geneva: World Health Organization; 1986. EPI/GEN/86/4 Rev 1.

10. Henderson $\mathrm{RH}$, Davis $\mathrm{H}$, Eddins DL, Foege $\mathrm{WH}$. Assessment of vaccination coverage, vaccination scar rates, and smallpox scarring in five areas of West Africa. Bull World Health Organ. 1973; 48:183-94.

11. Henderson RH, Sundaresan T. Cluster sampling to assess immunization coverage: a review of experience with simplified sampling method. Bull World Health Organ. 1982;60: 253-60.

12. Cutts FT. The use of the WHO cluster survey for evaluating the impact of the expanded programme on immunization on target disease incidence. J Trop Med Hyg. 1988;91:231-9.

13. Turner AG, Magnani RJ, Shuaib M. A not quite as quick but much cleaner alternative to the Expanded Programme on Immunization
(EPI) cluster survey design. Int J Epidemiol. 1996;25:198-203.

14. National Childhood Vaccine Injury Act: requirements for permanent vaccination records and for reporting of selected events after vaccination. MMWR. 1988;37:197-200.

15. Centers for Disease Control and Prevention Recommendations of the Advisory Committee on Immunization Practices: programmatic strategies to increase vaccination ratesassessment and feedback of provider-based vaccination coverage information. MMWR. 1996;45:219-20.

16. Lionis C, Chatziarsenis M, Antonakis N, Gianoulis Y, Fioretos M. Assessment of vaccine coverage of schoolchildren in three primary health care areas in rural Crete, Greece. Fam Pract. 1998;15:443-8.

17. Costa Rica. Proyecto Estado de la Nación. Estado de la Nación en desarrollo humano sostenible. San José, Costa Rica: Proyecto Estado de la Nación; 2001.

Manuscrito recibido el 28 de septiembre de 2003. Aceptado para publicación, tras revisión, el 1 de julio de 2004 
ABSTRACT Objective. To identify differences in the level of coverage of and opportunity for vaccination among schoolchildren in three areas in Costa Rica with different characteristics: an urban area (with the highest level of socioeconomic development of the three areas), a rural area (with a medium level of socioeconomic development), and a border area (a rural area in northern Costa Rica, on the border with Nicaragua, with the lowest level of socioeconomic development and the highest proportion of foreign immigrants).

Methodology. Following selection of schools by proportional probability, surveys were used with children chosen at random from the first and second grades of elementary schools in the three areas: urban (961 students), rural (544 students), and border (811 students). The data on the vaccines that had been administered were obtained from the children's vaccination cards. Differences among the three areas were evaluated: (1) in the coverage with BCG; with three doses of diphtheria-tetanuspertussis vaccine (DTP3); with three doses of oral polio vaccine (OPV3); with the first dose of measles-mumps-rubella vaccine (MMR1); and with the second dose of MMR vaccine (MMR2) and (2) in the "opportunity" for the children having received DTP1 + OPV1 before 3 months of age, DTP3 + OPV3 before 7 months of age, and DTP4 + OPV4 + MMR1 before 24 months of age.

Results. Out of all the students who had been selected, $80 \%$ of them in the urban area had a vaccination card, $73 \%$ did in the rural area, and $72 \%$ did in the border area $(P<$ 0.05). The coverage levels for BCG, DTP3, and OPV3 were each over $95 \%$ in both the urban area and the rural area; however, the coverage levels were significantly lower $(P<0.05)$ in the border area: BCG, 83\%; OPV3, 88\%; and DTP3, 88\%. Coverage with MMR1 and MMR2 was similar in the three areas. The percentage of schoolchildren with two or more doses of measles vaccine was $98 \%$ in the urban area, $92 \%$ in the rural area, and $85 \%$ in the border area $(P<0.05)$. In terms of opportunity, $90 \%$ of the children had received DTP1 + OPV1 before 3 months of age in the urban area, $89 \%$ had in the rural area, and $80 \%$ had in the border area $(P<0.05)$. The percentage of application of the complete basic schedule (DTP4 + OPV4 + MMR1) before 24 months of age was $93 \%$ in the urban area, 95\% in the rural area, and $84 \%$ in the border area $(P<0.05)$.

Conclusions. The border area had lower coverage of and opportunity for the basic schedule of vaccines, except for MMR. Follow-up campaigns for measles eradication have increased the coverage of the initial and booster doses in all three areas, but the increase has been greatest in the urban area. A greater effort should be made to identify children with an incomplete schedule of vaccinations, with priority going to areas that have a high proportion of immigrants.

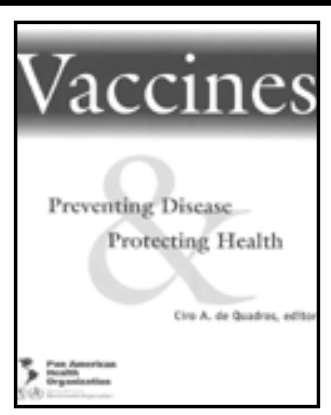

\section{Vaccines: Preventing Disease and Protecting Health IS NOW AVAILABLE IN ELECTRONIC FORMAT}

In this book you will find information about how vaccines have improved the health of the world's populations. The book relates successful efforts to fight disease with vaccines, including the eradication of polio from the Americas, and the potential contribution of new measles vaccine formulations to reducing measles mortality worldwide. It also looks at the challenges posed in using vaccines to cope with emerging and re-emerging diseases, such as HIV/AIDS, and in the fight against bioterrorism.

Electronic Version: 2004, 412p., ISBN 927511596 6, Order code: SP-E 596, Price: US $\$ 40.00$

To access the electronic version, follow these three simple steps:

1. Visit the site: www.ingentaselect.com

2. Select Pan American Health Organization in the Browse by publisher option

3. Select Vaccines: Preventing Disease and Protecting Health

Or go directly to PAHO publications by visiting:

http://www.ingentaselect.com/pubjournals.htm?pb=paho 